\title{
Avaliação da qualidade pós-colheita e conservação de cebolinhas submetidas ao hidroresfriamento
}

\section{Assessment of the post-harvest quality and conservation of spring onions submitted to hydrocooling}

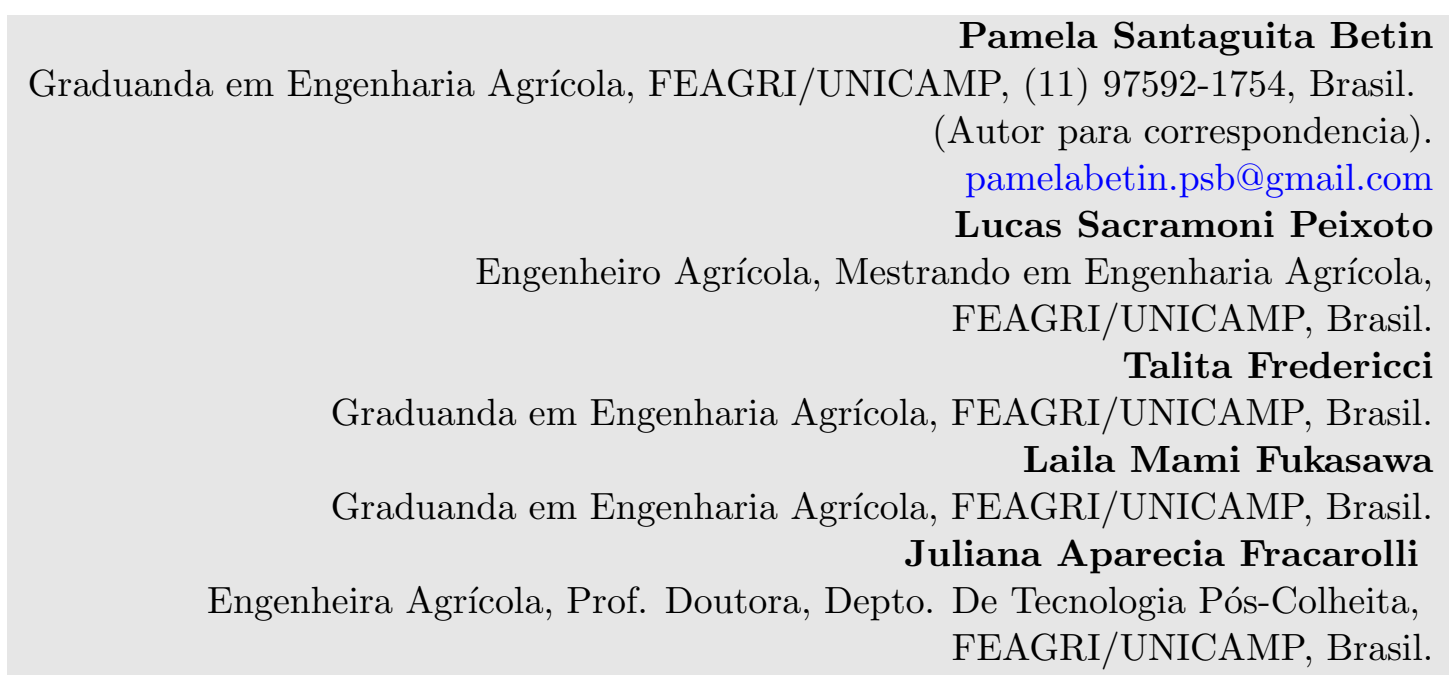

\section{Resumo}

A cebolinha pertencente à família das aliáceas (Alliaceae) é uma das hortaliças mais utilizadas e comercializadas do mundo, com carácter essencialmente condimentar. Entretanto, apresenta alta perecibilidade e suscetibilidade à perda de água, o que ocasiona redução da vida útil do produto, fazendo-se necessário o uso de técnicas de pós-colheita, com o intuito de diminuir a atividade metabólica e prolongar a vida útil do mesmo, tanto na prateleira (benefício ao produtor) como na geladeira (benefício ao consumidor). O método do hidroresfriamento que consiste na imersão do produto em uma mistura de água e gelo, se apresenta como uma boa alternativa para retirada do calor de campo e favorecimento da umidade dos produtos hortícolas, além de poder ser associado com o processo de sanitização dos mesmos. Diante do exposto, o presente trabalho teve por objetivo verificar a eficiência e os efeitos do hidroresfriamento na qualidade e conservação pós-colheita da cebolinha, através das análises de parâmetros de qualidade como perda de massa fresca, sólidos solúveis totais (SST), pH, acidez total titulável (ATT), índice de clorofila, relação SST/ATT, respiração, índice de escurecimento e biospeckle pelo método do momento de inércia, visando determinar o melhor tempo de aplicação do hidroresfriamento a $5{ }^{\circ} \mathrm{C}$. O hidroresfriamento se mostrou eficiente, principalmente, no controle de perda de massa e nos aspectos visuais comerciais, sendo o tempo mais indicado de aplicação o de 10 minutos.

Palavras-chave: pré-resfriamento; tratamento hidrotérmic; hortaliças; comercialização; biospeckle 


\begin{abstract}
Spring onions are part of the Alliaceae family and is one of the most used and traded vegetables in the world, with an essentially seasoning nature. However, they present high perishability and susceptibility to water loss, which reduces the useful life of the product, both on shelves (benefit for the producer) and in refrigerators (benefit for the consumer). The hydrocooling method, which consists of the immersion of the product in a mixture of water and ice, is a good alternative for field heat removal and favouring the humidity of vegetable products, besides being associated to its sanitation process. Considering what has been said, this paper aimed to check the efficiency and the effects of hydrocooling on the post-harvest quality and conservation of spring onions, through analyses of quality parameters such as fresh mass loss, total soluble solids (TSS), pH, total titratable acidity (TTA), chlorophyll index, TSS/TTA ratio, respiration, darkening index, and biospeckle through the moment of inertia method, aiming to determine the best hydrocooling application time at $5{ }^{\circ} \mathrm{C}$. Hydrocooling showed efficient, mainly on the mass loss control and on the commercial visual aspects, with 10 minutes as the most indicated application time.
\end{abstract}

Keywords: pre-cooling; hydrothermal treatment; vegetables; trade; biospeckle

\section{Introdução}

A cebolinha, pertencente à família das aliáceas Alliaceae é uma planta essencialmente condimentar e amplamente cultivada no país, estando presente em muitos lares brasileiros. Devido a sua alta perecibilidade e suscetibilidade à perda de água, intensificada pelo manejo inadequado de temperatura e umidade do ar nos locais de armazenamento, faz-se necessário o uso de técnicas pós-colheita, com o intuito de diminuir a atividade metabólica e prolongar a vida útil do produto, tanto na prateleira (benefício ao produtor) como na geladeira (benefício ao consumidor) (Souza, et al., 2017). Produtos insensíveis ao excesso de umidade, ou que não tem suas características influenciadas pelas mesmas, como por exemplo: melão, kiwi, alho-poró, espinafre, milho, entre outros, inclusive a cebolinha, podem utilizar o método de hidroresfriamento com água refrigerada como alternativa de tratamento pós-colheita, que se destaca entre várias técnicas por sua simplicidade, praticidade, eficiência e baixo custo (Kanlayanarat \& Acedo, 2009).

O método do hidroresfriamento, que consiste na aspersão de água sobre o produto, ou imersão do mesmo em água gelada (mistura de água e gelo), logo após a sua retirada do campo e antes de realizar a sua embalagem, armazenagem e/ou distribuição se apresenta como uma boa alternativa (simples, eficiente e de baixo custo) na conservação de produtos hortícolas, pois reduz o calor de campo e diminui os processos metabólicos, responsáveis pela senescência, reduzindo também a perda de umidade (Teixeira, et al., 2016). Entretanto, segundo Silva et al (2015), em relação às cebolinhas, são poucos os trabalhos encontrados na literatura dedicados a avaliar suas qualidades físico-químicas, estabilidade do produto, ou até mesmo sobre os tratamentos pós-colheita.

Diante do exposto, este trabalho tem por objetivo verificar a eficiência e os efeitos do hidroresfriamento na qualidade e conservação pós-colheita da cebolinha, através das análises de parâmetros de qualidade como perda de massa fresca, sólidos solúveis totais, $\mathrm{pH}$, acidez total titulável, índice de clorofila, relação SST/ATT, e determinar o melhor tempo de hidroresfriamento a $5{ }^{\circ} \mathrm{C}$.

\section{Material e métodos}

O experimento foi realizado entre os dias 18 e 26 de julho de 2017 no Laboratório de Tecnologia em Pós-colheita (LTPC), da Faculdade de Engenharia Agrícola (FEAGRI) - Universidade Estadual de Campinas (UNICAMP) - campus Campinas SP, sendo utilizados aproximadamente $15 \mathrm{~kg}$ de cebolinha comum (Allium fistulosum).

O pré-resfriamento foi realizado por imersão em 
gelo moído e água na proporção de $1 / 3\left(\mathrm{v} \mathrm{v}^{-1}\right)$, mantendo uma temperatura de $5{ }^{\circ} \mathrm{C}$ com um auxílio de um termômetro. Sendo 5 tratamentos distintos: Tratamento 1 (T1) - Testemunha: sem hidroresfriamento (imersão em água destilada com sanitizante a temperatura ambiente apenas para lavagem); Tratamento 2 (T2) - Hidroresfriamento a $5{ }^{\circ} \mathrm{C}$ por $5 \mathrm{~min}$; Tratamento 3 (T3) Hidroresfriamento a $5{ }^{\circ} \mathrm{C}$ por $10 \mathrm{~min}$; Tratamento 4 (T4) - Hidroresfriamento a $5{ }^{\circ} \mathrm{C}$ por $15 \mathrm{~min}$; e Tratamento 5 (T5) - Hidroresfriamento a $5{ }^{\circ} \mathrm{C}$ por 20 min. Após a aplicação as amostras foram centrifugadas e armazenadas em geladeira.

Foram realizadas as análises físico químicas de perda de massa fresca, sólidos solúveis totais (SST), acidez total titulável (ATT), índice de clorofila, taxa respiratória, cor, biospeckle pelo método do momento de inércia e visuais quanto a qualidade (verificando-se a ocorrência de sintomas indicativos de perda de qualidade).

A Acidez Total Titulável (em \% de ácido por $100 \mathrm{~g}$ ) foi determinada a partir de titulação com $\mathrm{NaOH} 0.1 \mathrm{M}$ de solução, através da homogeneização de $10 \mathrm{~g}$ da folha macerada diluída em $90 \mathrm{~mL}$ de água destilada, e pela equação (1) (Carvalho, et al., 1990).

$$
\operatorname{ATT}\left(\frac{g}{100 g}\right)=\frac{n * N * E q}{10 * p}
$$

em que:

$\mathrm{N}=$ normalidade da solução de $\mathrm{NaOH}$;

$\mathrm{n}=$ volume da solução de $\mathrm{NaOH}$ gastos na titulação em $\mathrm{mL}$;

$\mathrm{p}=$ massa da amostra em gramas;

$\mathrm{V}=$ volume da amostra em $\mathrm{mL}$;

$\mathrm{Eq}=$ equivalente-grama do ácido.

A avaliação de sólidos solúveis totais foi realizada por leitura direta em refratômetro digital Reichert automático ABBE MARK III com resultados expressos em ${ }^{\circ}$ Brix. A relação SST/ATT foi calculada pela divisão direta dos valores dos parâmetros de ${ }^{\circ}$ Brix pelos valores de percentual de ácido (ATT) (Petry, et al., 2012). O Índice de Clorofila foi obtido a partir de método não destrutivo, utilizando medidor de clorofila SPAD (Soil Plant Analysis Development Section, Minolta) (Oliveira, 2012).

As medições da taxa respiratória foram realizadas utilizando um analisador de composição gasosa (CO2 e O2) Pac Check 325 da marca MOCON. As amostras foram colocadas em um recipiente de vidro hermeticamente fechado para posterior acondicionamento em geladeira a $10{ }^{\circ} \mathrm{C}$ por 30 minutos. Após este tempo foram obtidas as $\%$ de $\mathrm{CO}_{2}$, para o cálculo das taxas em mg $\mathrm{CO}_{2} \mathrm{~kg}^{1}$ $\mathrm{h}^{1}$, segundo a equação (2).

$$
P_{\mathrm{CO}_{2}}=\frac{\% \mathrm{CO}_{2}}{100} * \frac{V_{\text {vazios }}}{m_{p} \text { roducto }} * \frac{1,98}{t}
$$

em que:

\%CO2 = Produção de CO2 pelo produto em \%;

$\mathrm{V}_{\text {vazios }}=$ Volume de vazios no recipiente, $\mathrm{em} \mathrm{mL}$;

$\mathrm{m}_{\text {produto }}=$ Massa de produto no recipiente, em kg;

$\mathrm{t}=$ Tempo em que os recipientes ficaram fechados em horas.

O Índice de Escurecimento (IE) foi determinado a partir da utilização colorímetro portátil do modelo mini Scan XE da marca Hunter e realizada a leitura direta dos parâmetros de Hunter, $L^{*}, a^{*}$ e b* (Bible \& Singha, 1993) e calculado de acordo com Palou et al (1999), conforme equação (3) e (4):

$$
\begin{gathered}
I E=\frac{[100 *(X-0,31)]}{0,172} \\
X=\frac{(a+1,75 L)}{(5,645 L+a-3,02 b)}
\end{gathered}
$$

em que:

$\mathrm{L}=$ luminosidade $(0=$ preto, $100=$ branco $)$;

$\mathrm{a}=$ coordenada de $\operatorname{croma}(-\mathrm{a}=$ verde,$+\mathrm{a}=$ vermelho);

$\mathrm{b}=$ coordenada de croma $(-\mathrm{b}=$ azul e $+\mathrm{b}=$ amarelo). 
Nos ensaios de Biospeckle (Figura 1) foram utilizados: um laser vermelho de diodo de $632 \mathrm{~nm}$ de comprimento de onda e $10 \mathrm{~mW}$ de potência ligado a uma fonte (bateria), um celular smartphone com câmera filmadora digital, um computador com softwares para o processamento das imagens e para tratamento estatístico. Para o processamento das imagens foram usados os softwares Matlab e ImageJ versão 1.50i (Schneider \& Rasband, 2012).

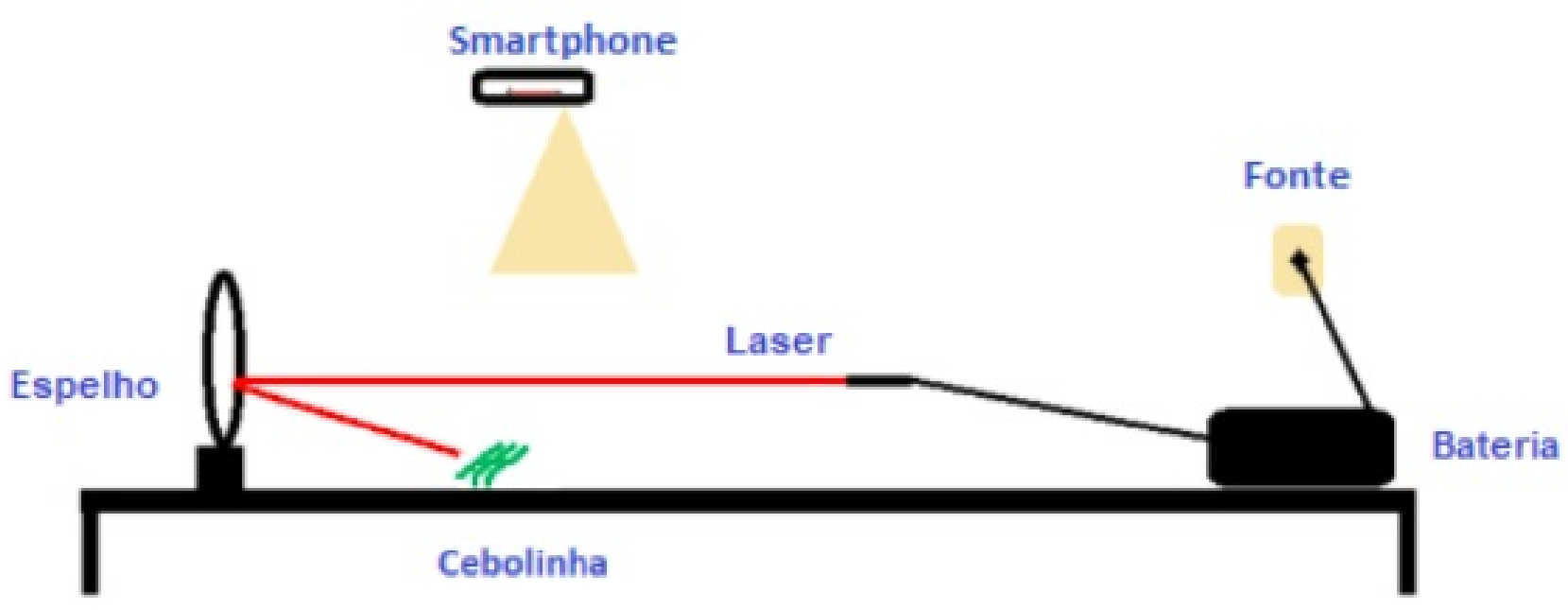

Figura 1. Configuração experimental para avaliação através do Biospeckle.

\section{Resultados e discussão}

Ao longo do período de armazenamento o teor de sólidos solúveis nas amostras aumentou (Tabela 1), o que pode ser explicado pela perda de água pelo produto. Silva et al (2015) encontrou para a cebolinha comum, logo após a colheita, 4.65 de ${ }^{\circ}$ Brix, valor este próximo do obtido neste trabalho para o dia 1 de armazenamento.

Tabela 1. Resultados dos valores médios, em ${ }^{\circ}$ Brix, dos sólidos solúveis totais

\begin{tabular}{lllllll}
\hline Tratamento & Dia 1 & Dia 3 & Dia 4 & Dia 5 & Dia 7 & Dia 9 \\
\hline Testemunha & $5.04^{a 2}$ & $4.11^{a 3}$ & $5.79^{a 4}$ & $5.04^{a 3}$ & $4.83^{a 1}$ & $6.20^{a 2}$ \\
\hline 5 min & $4.54^{a 1 a 2}$ & $3.64^{a 2}$ & $4.14^{a 2}$ & $3.75^{a 1 a 2}$ & $4.73^{a 1}$ & $4.77^{a 1}$ \\
\hline $10 \mathrm{~min}$ & $4.75^{a 2}$ & $3.25^{a 1}$ & $3.69^{a 1}$ & $3.57^{a 1 a 2}$ & $5.12^{a 1 a 2}$ & $6.56^{a 2}$ \\
\hline $15 \mathrm{~min}$ & $3.74^{a 1}$ & $4.14^{a 3}$ & $3.72^{a 1}$ & $3.96^{a 2}$ & $5.49^{a 2}$ & $5.23^{a 1}$ \\
\hline $20 \mathrm{~min}$ & $4.54^{a 1 a 2}$ & $3.57^{a 2}$ & $4.67^{a 3}$ & $3.12^{a 1}$ & $4.69^{a 1}$ & $6.39^{a 2}$ \\
\hline
\end{tabular}

*Médias seguidas pela mesma letra e número, dentro de cada tratamento, não diferem entre si, estatisticamente, pelo teste de Tukey a $5 \%$ de probabilidade.

A partir do $5^{\circ}$ dia de armazenamento os teores de $\mathrm{pH}$ (Tabela 2) apresentaram tendência de crescimento, apesar de oscilarem até esse momento, comportamento também observado por Souza et al (2017) no hidroresfriamento aplicado a coentros, e explicado por Chitarra \& Chitarra (2007) que diz que o aumento ocorre devido às altas taxas de respiração que os produtos hortícolas realizam para se manterem vivos. 
Tabela 2. Resultados dos valores médios de pH para os diferentes tratamentos.

\begin{tabular}{lllllll}
\hline Tratamento & Dia 1 & Dia 3 & Dia 4 & Dia 5 & Dia 7 & Dia 9 \\
\hline Testemunha & $5.56^{a 1}$ & $5.62^{a 1}$ & $5.71^{a 2}$ & $5.53^{a 1}$ & $5.69^{a 1}$ & $5.70^{a 1}$ \\
\hline $5 \mathrm{~min}$ & $5.29^{a 1}$ & $5.62^{a 1}$ & $5.59^{a 1}$ & $5.50^{a 1}$ & $5.66^{a 1}$ & $5.74^{a 1 a 2}$ \\
\hline $10 \mathrm{~min}$ & $5.31^{a 1}$ & $5.74^{a 2}$ & $5.64 a 1^{a 2}$ & $5.60^{a 1 a 2}$ & $5.69^{a 1}$ & $5.72^{a 1 a 2}$ \\
\hline $15 \mathrm{~min}$ & $5.56^{a 1}$ & $5.65^{a 1 a 2}$ & $5.59^{a 1}$ & $5.60^{a 1 a 2}$ & $5.73^{a 1 a 2}$ & $5.77^{a 1 a 2}$ \\
\hline $20 \mathrm{~min}$ & $5.53^{a 1}$ & $5.68^{a 1 a 2}$ & $5.69^{a 2}$ & $5.67^{a 2}$ & $5.78^{a 2}$ & $5.79^{a 2}$ \\
\hline
\end{tabular}

*Médias seguidas pela mesma letra e número, dentro de cada tratamento, não diferem entre si, estatisticamente, pelo teste de Tukey a $5 \%$ de probabilidade.

Para a acidez total titulável (Tabela comportamento evidente. Entretanto, vale destacar 3) observou-se que os valores apresentaram que o tratamento T4 apresentou-se no geral com os variabilidade ao longo do período para todos os valores mais baixos e próximos do valor médio de tratamentos, com altas e baixas ao longo do período 0.09-100 g-1 encontrado para cebolinha comum por de armazenamento, não havendo um padrão de Silva et al (2015).

Tabela 3. Resultados dos valores médios de Acidez total titulável para os diferentes tratamentos

\begin{tabular}{lllllll}
\hline Tratamento & Dia 1 & Dia 3 & Dia 4 & Dia 5 & Dia 7 & Dia 9 \\
\hline Testemunha & $0.119^{a 2 a 3}$ & $0.103^{a 2}$ & $0.086^{a 1}$ & $0.131^{a 3}$ & $0.113^{a 1}$ & $0.151^{a 2}$ \\
\hline 5 min & $0.119^{a 2}$ & $0.081^{a 1}$ & $0.09^{a 1}$ & $0.103^{a 2}$ & $0.105^{a 1}$ & $0.118^{a 1}$ \\
\hline $10 \mathrm{~min}$ & $0.118^{a 2}$ & $0.087^{a 1 a 2}$ & $0.082^{a 1}$ & $0.098^{a 1 a 2}$ & $0.115^{a 1}$ & $0.13^{a 1 a 2}$ \\
\hline $15 \mathrm{~min}$ & $0.089^{a 1}$ & $0.085^{a 1 a 2}$ & $0.092^{a 1}$ & $0.079^{a 1}$ & $0.086^{a 1}$ & $0.108^{a 1}$ \\
\hline $20 \mathrm{~min}$ & $0.139^{a 3}$ & $0.076^{a 1}$ & $0.099^{a 1}$ & $0.088^{a 1 a 2}$ & $0.118^{a 1}$ & $0.116^{a 1}$ \\
\hline
\end{tabular}

* Médias seguidas pela mesma letra e número, dentro de cada tratamento, não diferem entre si, estatisticamente, pelo teste de Tukey a $5 \%$ de probabilidade.

Para os valores de índice de escurecimento armazenamento todas as amostras sofreram "perda (Tabela 4) não foi observada diferença significativa de cor", ficando em tons de verde mais claro, sendo entre os tratamentos, entretanto vale destacar que o mesmo observado por Nascimento, et al., (2017) comparando o primeiro com o último dia de ao aplicar tratamento hidrotérmico em alfaces.

Tabela 4. Resultados dos valores médios de Índice de escurecimento para os diferentes tratamentos

\begin{tabular}{lllllll}
\hline Tratamento & Dia 1 & Dia 3 & Dia 4 & Dia 5 & Dia 7 & Dia 9 \\
\hline Testemunha & $29.94^{a 1}$ & $24.85^{a 1}$ & $18.91^{a 1}$ & $32.06^{a 1}$ & $33.56^{a 1}$ & $19.89^{a 1}$ \\
\hline 5 min & $26.82^{a 1}$ & $26.21^{a 1}$ & $19.57^{a 1}$ & $31.50^{a 1}$ & $30.60^{a 1}$ & $15.04^{a 1}$ \\
\hline $10 \min$ & $29.68^{a 1}$ & $24.62^{a 1}$ & $16.81^{a 1}$ & $31.88^{a 1}$ & $29.21^{a 1}$ & $15.26^{a 1}$ \\
\hline $15 \min$ & $28.04^{a 1}$ & $27.57^{a 1}$ & $18.37^{a 1}$ & $26.69^{a 1}$ & $25.92^{a 1}$ & $15.25^{a 1}$ \\
\hline 20 min & $31.40^{a 1}$ & $26.52^{a 1}$ & $20.11^{a 1}$ & $31.05^{a 1}$ & $30.79^{a 1}$ & $16.40^{a 1}$ \\
\hline
\end{tabular}

*Médias seguidas pela mesma letra e número, dentro de cada tratamento, não diferem entre si, estatisticamente, pelo teste de Tukey a $5 \%$ de probabilidade. 
A testemunha foi a que apresentou a menor vida útil com índice de maturação no $4^{\mathrm{O}}$ dia de armazenamento (Tabela 5), sendo este segundo Sanches, et al., (2015) o momento de aceleração do metabolismo promovido pelo consumo de açúcares associado à concentração dos ácidos orgânicos, levando à senescência com média de 67.08 e redução gradual até o fim da análise. Por sua vez as amostras do T3 apresentaram maior vida útil tendo seu índice de qualidade até o $9^{\circ}$ dia com 50.67.

Tabela 5. Resultados dos valores médios da relação Sólidos solúveis pela acidez total titulável

\begin{tabular}{lllllll}
\hline Tratamento & Dia 1 & Dia 3 & Dia 4 & Dia 5 & Dia 7 & Dia 9 \\
\hline Testemunha & $42.19^{a 1}$ & $39.85^{a 1 a 2}$ & $67.08^{a 2}$ & $38.29^{a 1}$ & $43.25^{a 1}$ & $40.97^{a 1 a 2}$ \\
\hline $5 \mathrm{~min}$ & $38.01^{a 1}$ & $45.73^{a 1 a 2}$ & $45.85^{a 1}$ & $36.18^{a 1}$ & $45^{a 1}$ & $40.34^{a 1}$ \\
\hline $10 \mathrm{~min}$ & $39.99^{a 1}$ & $37.11^{a 1}$ & $44.91^{a 1}$ & $36.40^{a 1}$ & $44.34^{a 1}$ & $50.67^{a 2 a 3}$ \\
\hline $15 \mathrm{~min}$ & $41.82^{a 1}$ & $48.79^{a 2}$ & $40.55^{a 1}$ & $50.64^{a 2}$ & $66.05^{a 2}$ & $48.40^{a 1 a 2 a 3}$ \\
\hline $20 \mathrm{~min}$ & $32.73^{a 1}$ & $46.79^{a 1 a 2}$ & $47.33^{a 1}$ & $35.23^{a 1}$ & $39.45^{a 1}$ & $54.87^{a 3}$ \\
\hline
\end{tabular}

*Médias seguidas pela mesma letra e número, dentro de cada tratamento, não diferem entre si, estatisticamente, pelo teste de Tukey a $5 \%$ de probabilidade.

Apesar de esperar-se que as taxas de respiração (2004), não foram evidenciadas diferenças sofressem redução para as amostras submetidas significativas entre as taxas de respiração obtidas ao hidroresfriamento conforme observado por para os tratamentos neste trabalho (Tabela 6). Travassos, et al., (2017) e por Kalbasi-Ashtari

Tabela 6. Resultados dos valores médios das Taxas de respiração em mg de $\mathrm{CO} 2 \mathrm{~kg}^{-1} \mathrm{~h}^{-1}$

\begin{tabular}{lllllll}
\hline Tratamento & Dia 1 & Dia 3 & Dia 4 & Dia 5 & Dia 7 & Dia 9 \\
\hline Testemunha & $337.86^{a 1}$ & $276.01^{a 1}$ & $490.03^{a 1}$ & $514.74^{a 1}$ & $633.65^{a 1}$ & $694.93^{a 1}$ \\
\hline 5 min & $391.27^{a 1}$ & $372.12^{a 1}$ & $653.45^{a 1}$ & $514.71^{a 1}$ & $710.68^{a 1}$ & $559.71^{a 1}$ \\
\hline $10 \mathrm{~min}$ & $387.55^{a 1}$ & $367.84^{a 1}$ & $695.37^{a 1}$ & $559.80^{a 1}$ & $622.29^{a 1}$ & $548.64^{a 1}$ \\
\hline $15 \mathrm{~min}$ & $344.47^{a 1}$ & $372^{a 1}$ & $594.65^{a 1}$ & $569.86^{a 1}$ & $587.49^{a 1}$ & $602.57^{a 1}$ \\
\hline $20 \mathrm{~min}$ & $368.56^{a 1}$ & $368.09^{a 1}$ & $560.17^{a 1}$ & $586.53^{a 1}$ & $660.73^{a 1}$ & $629.32^{a 1}$ \\
\hline
\end{tabular}

Médias seguidas pela mesma letra e número, dentro de cada tratamento, não diferem entre si, estatisticamente, pelo teste de Tukey a $5 \%$ de probabilidade.

Os valores de perda de massa acumulada ao longo dos dias de armazenamento (Figura 2), evidenciam a eficiência do hidroresfriamento aplicado às cebolinhas no controle da perda de massa, sendo o tratamento com 10 min de aplicação a melhor opção com perda de massa total acumulada de $16.01 \%$, resultado quase $10 \%$ menor do que a amostra sem tratamento, fato também observado por Travassos, et al., (2017). 


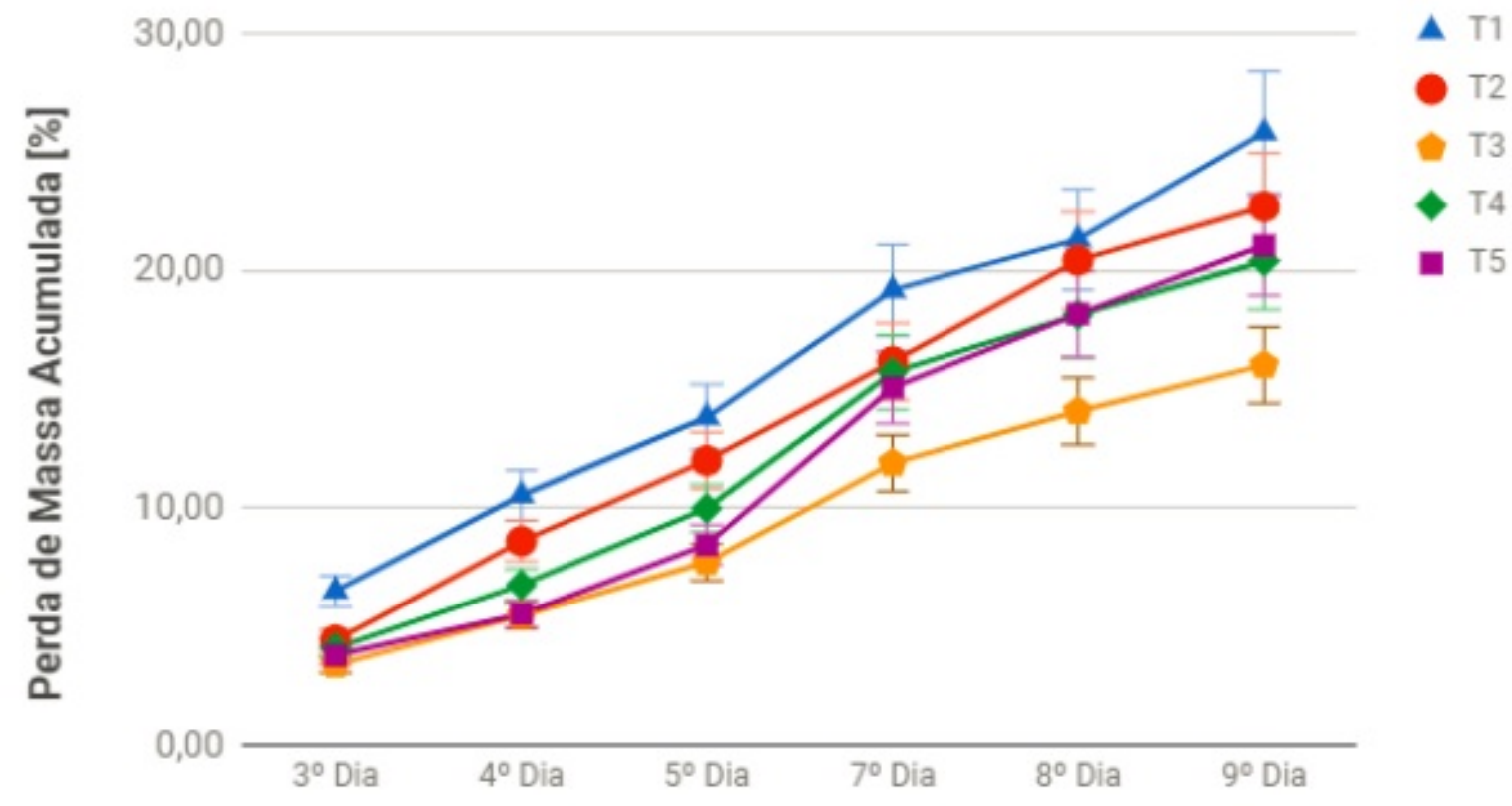

\section{Dias de Armazenamento}

Figura 2. Perda de massa acumulada em cebolinhas sob diferentes tratamentos

A perda de massa nas hortaliças folhosas gera queda acelerada da qualidade, sendo o murchamento e o enrugamento os principais indicativos da perda de água, podendo acelerar a deterioração pelo aumento da taxa de reações (Travassos, et al., 2017). Foram observadas mudanças significativas nos aspectos visuais das cebolinhas quanto ao murchamento e ao enrugamento, tendo as amostras T3 se mantido atraentes até o último dia de análise (Figura 3). Entretanto, não houve diferença significativa na cor das amostras, conforme os índices de clorofila e também observado por Travassos et al (2017).

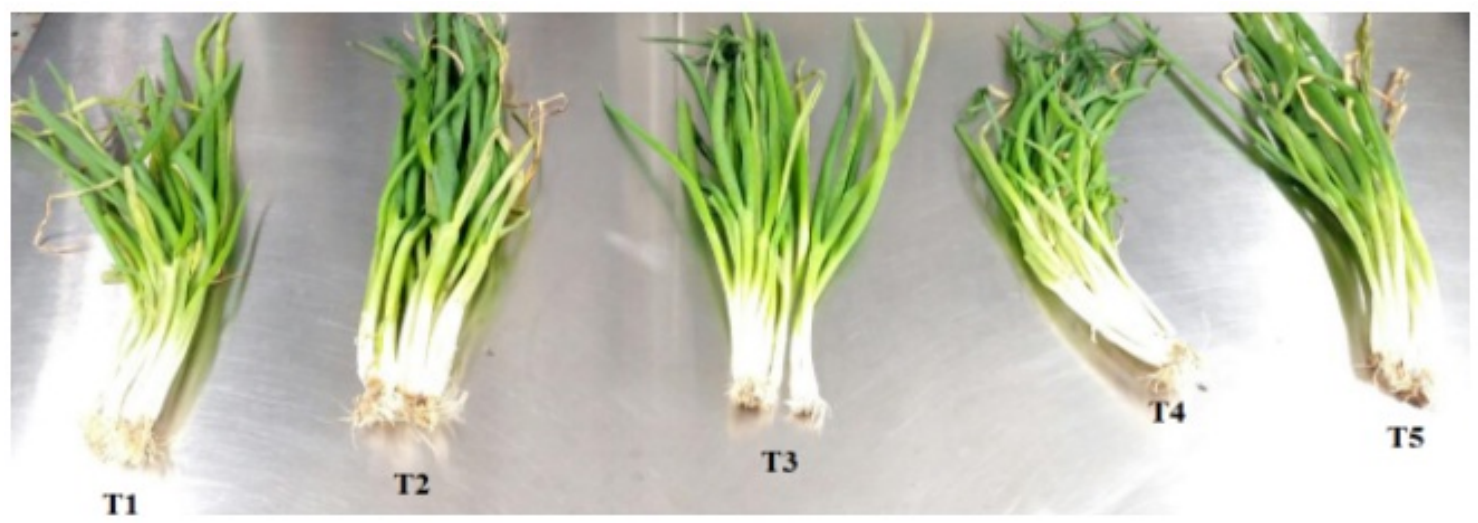

Figura 3. Aspecto visual das amostras sob diferentes tratamentos aos 9 dias de armazenamento

\section{Conclusões}

O hidroresfriamento se mostrou eficiente na conservação pós colheita de cebolinhas principalmente no controle de perda de massa e nos aspectos visuais comerciais, sendo o tempo mais indicado de hidroresfriamento o de 10 minutos.

\section{Referências bibliográficas}

Bible, B. B., Singha, S., 1993. Canopy position influences CIELAB coordinates of peach color. HortScience, v. 28, n. 10, p. 992-993. Consultado em 11 de fevereiro de 2019. 
https://eurekamag.com/research/002/315/0023159 56.php. DOI: https://doi.org/10.21273/hortsci.28.1 0.9922315956.php

Carvalho, C. R. L., Mantovani, D. M. B., Carvalho, P. R. N., Moraes, R. M. M., 1990. Análises químicas de alimentos. Campinas: ITAL, 121p.

Chitarra, M. I. F., Chitarra, A. B., 2007. Processamento mínimo de alface. In: MORETTI, C. L. (Ed.). Manual de Processamento Mínimo de Frutas e Hortaliças. Brasília: SEBRAE. p. 299-342. DOI: https://doi.org/10.26512/2007.03.tcc.165

Kalbasi-Ashtari, A., 2004. Effects of postharvest pre-colling processes and cyclical treatment on the physic. Chemical properties of " ked haven perchs" and "Shahmavch Pears" during cold storage. Agricultural engineering international: the CIGR journal of a scientific. Research and development, Florida, v.6.

Kanlaynarat, R., Acedo, Jr., 2009. Horticultural chain management for countries of Asia and pacific region: a training package. food and agriculture organizations of the united nations. Regional Office for Asia and The Pacific. Bangkok.

Nascimento, G. A. S., Sanches, G. A., Moreira, S. G. E., Cordeiro, M. A. C., 2017. Tratamento hidrotérmico na -conservação e qualidade pós-colheita de alface. Revista Trópica - Ciências Agrárias e Biológicas. p.65-76, v.09, n.01, 2017. ISSN 1982-4881. Consultado em 11 de fevereiro de 2019. http://www.periodicoseletronicos.ufma.br/index.ph $\mathrm{p} /$ ccaatropica/article/view/3454/4115

Oliveira, L. S., 2012. Efeito do hidroresfriamento, da temperatura e da rehidratação na conservação pós-colheita do coentro.Viçosa, MG - Brasil. Consultado em 11 de fevereiro de 2019. http://www.scielo.br/scielo.php?pid=S0102-053620 $15000400448 \&$ script $=$ sci_abstract\&tlng $=$ pt. $\quad$ DOI: https://doi.org/10.1590/s0102-053620150000400007

Palou, E., López-Malo, A., Barbosa-Cánovas, G. V., Welti-Chanes,, J., Swanson, B. G., 1999.
Polyphenoloxidase activity and color of blanched and high hydrostatic pressure treated banana puree. Journal of Food Science, v. 64, n. 1, p. 42-45. DOI: https://doi.org/10.1111/j.1365-2621.1999.tb09857.x

Petry, H. B., Koller, C. O., Bender, J. R., Schwarz, S. F., 2012. Qualidade de Laranjas 'valência' produzidas sob sistemas de cultivo orgânico e convencional. Rev. Bras. Frutic., Jaboticabal - SP, v. 34, n. 1, p. 167-174. Consultado em 11 de fevereiro de 2019. http://www.scielo.br/pdf/rbf/v34n1/v34n1a23.pdf. DOI: https://doi.org/10.1590/s0100-2945201200010 0023

Sanches, G. A., Silva, B. M., Moreira, S. E. G., Cordeiro, M. C. A., 2015. Comportamento fisiológico pós-colheita de cultivares de rúcula minimamente processadas. Acta Iguazu, Cascavel, v.4, n.1, p. 91-105. ISSN: 2316-4093. Consultado em 11 de fevereiro de 2019. http://saber.unioeste.br/index.php/actaiguazu/arti cle/view/13281

Schneider, C. A., Rasband, W. S., Eliceiri, K. W., 2012. NIH Image to ImageJ: 25 Years of Image Analysis. Nat. Methods 9: 671-5. Consultado em 11 de fevereiro de 2019. https://www.ncbi.nlm.nih.gov/pubmed/22930834. DOI: https://doi.org/10.1038/nmeth.2089

Silva, A. P. G., Borges C. D., Miguel, A. C. A., Jacomino, P. A., Mendonça, B. R. C., 2015. Características físico-químicas de cebolinhas comum e europeia. Campinas, v. 18 , n. 4, p. 293-298, out./dez. DOI: http://dx.doi.org/10.1590/1981-6723.3015

Souza, M. T. A., Sanches, A. G., Moreira, S. G. E., Cordeiro, C. A. M., 2017. Eficiência do hidroresfriamento na conservação e qualidade pós-colheita de coentro (Coriandrum sativum L.). Revista Trópica - Ciências Agrárias e Biológicas. p.32-40, v.10, n.01, ISSN 1982-4881. Consultado em 11 de fevereiro de 2019. http://www.periodicoseletronicos.ufma.br/index.ph p/ccaatropica/article/view/3451. DOI: https://doi. org/10.5935/paet.v10.n3.04 
Teixeira, D. A., Gomes, J. A. O., Bonfim, F. P. G., Pardo, P.I., Mayobre, M. T.,2016. Técnicas de conservação pós-colheita para o manjericão. Rev. Bras. Pl. Med., Campinas, v.18, n.1, p.168-171, DOI: http://dx.doi.org/10.1590/1983-084X/15_007
Travassos, A. P., Silva, N. E., Pedroza, C. R. R., Soares, M. C. R. D., 2017. Hidroresfriamento na conservação pós-colheita de cebolinha. Revista Brasileira de Agropecuária Sustentável (RBAS), v.7, n.2, p.46-51. DOI: 10.21206/rbas.v7i2.396.

La Revista Ingeniería y Región cuenta con la Licencia Creative Commons Atribución (BY), No Comercial (NC) y Compartir Igual (SA)

(c) (i) (S) (O) 